\title{
OPTICAL SPECTRA OF CANDIDATE INTERNATIONAL CELESTIAL REFERENCE FRAME (ICRF) FLAT-SPECTRUM RADIO SOURCES
}

\author{
O. Titov $^{1}$, Laura M. Stanford ${ }^{1}$, Helen M. Johnston ${ }^{2}$, T. Pursimo ${ }^{3}$, Richard W. Hunstead ${ }^{2}$, \\ David L. JaunceY ${ }^{4}$, K. Maslennikov ${ }^{5}$, and A. BoldycheVa ${ }^{6}$ \\ ${ }^{1}$ Geoscience Australia, P.O. Box 378, Canberra, ACT 2601, Australia; oleg.titov@ga.gov.au \\ ${ }^{2}$ Sydney Institute for Astronomy, School of Physics, University of Sydney, NSW 2006, Australia \\ ${ }^{3}$ Nordic Optical Telescope, Nordic Optical Telescope Apartado 474E-38700 Santa Cruz de La Palma, Santa Cruz de Tenerife, Spain \\ ${ }^{4}$ CSIRO Astronomy and Space Science, ATNF \& Research School of Astronomy \& Astrophysics, Australian National University, \\ Canberra, ACT 2611, Australia \\ 5 The Central Astronomical Observatory at Pulkovo, Pulkovskoye Shosse, 65/1, 196140, St. Petersburg, Russia \\ ${ }^{6}$ Ioffe Physical Technical Institute, 26 Polytekhnicheskaya, St. Petersburg, 194021, Russia \\ Received 2013 February 18; accepted 2013 May 1; published 2013 June 10
}

\begin{abstract}
Continuing our program of spectroscopic observations of International Celestial Reference Frame (ICRF) sources, we present redshifts for 120 quasars and radio galaxies. Data were obtained with five telescopes: the $3.58 \mathrm{~m}$ European Southern Observatory New Technology Telescope, the two $8.2 \mathrm{~m}$ Gemini telescopes, the $2.5 \mathrm{~m}$ Nordic Optical Telescope (NOT), and the $6.0 \mathrm{~m}$ Big Azimuthal Telescope of the Special Astrophysical Observatory in Russia. The targets were selected from the International VLBI Service for Geodesy \& Astrometry candidate International Celestial Reference Catalog which forms part of an observational very long baseline interferometry (VLBI) program to strengthen the celestial reference frame. We obtained spectra of the potential optical counterparts of more than 150 compact flat-spectrum radio sources, and measured redshifts of 120 emission-line objects, together with $19 \mathrm{BL}$ Lac objects. These identifications add significantly to the precise radio-optical frame tie to be undertaken by Gaia, due to be launched in 2013, and to the existing data available for analyzing source proper motions over the celestial sphere. We show that the distribution of redshifts for ICRF sources is consistent with the much larger sample drawn from Faint Images of the Radio Sky at Twenty cm (FIRST) and Sloan Digital Sky Survey, implying that the ultra-compact VLBI sources are not distinguished from the overall radio-loud quasar population. In addition, we obtained NOT spectra for five radio sources from the FIRST and NRAO VLA Sky Survey catalogs, selected on the basis of their red colors, which yielded three quasars with $z>4$.
\end{abstract}

Key words: BL Lacertae objects: general - galaxies: active - quasars: emission lines - radio continuum: general reference systems

Online-only material: color figures, extended figure, machine-readable and VO tables

\section{INTRODUCTION}

The coming astrometric space mission, Gaia (Perryman et al. 2001; Mignard \& Klioner 2012), to be launched in 2013 by the European Space Agency, will measure high precision positions $(\sim 26 \mu$ as for $V=15 \mathrm{mag}, \sim 300 \mu$ as for $V=20 \mathrm{mag}$; de Bruijne 2012) and proper motions of $\sim 500,000$ quasars brighter than $m_{v}=20$. This new optical astrometric catalog will be linked with the current radio astrometric catalog, ICRF2, the second realization of the International Celestial Reference Frame (Fey et al. 2009). Optical counterparts of the extragalactic sources are being sought to confirm their identification as quasars.

This paper is the second in our series aimed at investigating the optical spectra of radio sources in the International VLBI Service for Geodesy \& Astrometry (IVS) Reference Catalog; see Schlüter \& Behrend (2007) for a description of the IVS history. Astrometric very long baseline interferometry (VLBI) measures the differences in arrival times of radio waves from ultracompact, flat-spectrum radio sources at telescopes positioned large distances apart. This procedure determines the positions of such sources to milliarcsecond precision.

The International Celestial Reference System (ICRS; Arias et al. 1995) was adopted by the International Astronomical Union as a reference system with its origin at the barycentre of the solar system (MacCarthy \& Petit 2004), and axes fixed by the positions of selected extragalactic radio sources. The first realization of the International Celestial Reference Frame (ICRF1) was used to establish the orientation of the ICRS axes (Ma et al. 1998). The current International Celestial Reference Frame, known as ICRF2, is based on a catalog of 295 "defining" sources. The formal weighted errors in the radio positions are reported by Fey et al. (2009) to have an upper limit to the noise floor of $41 \mu$ as.

The IVS astrometric program has a total catalog of $>6000$ radio sources, where $\sim 1200$ are observed on a regular basis. In the Southern Hemisphere, there is a significant deficit in candidate sources as well as a lack of optical identifications. By 2012 February, of the 3257 objects with measured redshifts, only 1213 are in the southern hemisphere and only 287 have declinations south of $-40^{\circ}$. This paucity of redshifts in the south leads to problems in the analysis of apparent proper motions of the reference radio sources (Titov \& Malkin 2009). To address this issue, an extensive program was started in 2010 to find optical counterparts and determine redshifts for southern IVS sources (Titov et al. 2011).

The quasars at high redshift $(z \geqslant 2)$ will be used for more intensive observations at the VLBI facilities in the southern hemisphere. VLBI observations of weak sources will be undertaken with the $64 \mathrm{~m}$ telescope in Parkes, Australia, and two $26 \mathrm{~m}$ telescopes in Hobart (Australia) and HartRAO (South Africa). Stronger radio sources (flux density $\geqslant 400 \mathrm{mJy}$ ) will 
be monitored with four $12 \mathrm{~m}$ telescopes recently installed in Australia: the AuScope network comprising Hobart, Yarragadee, and Katherine in Australia (Titov et al. 2013), and Warkworth in New Zealand. Several quasars found during the first observing run with the New Technology Telescope (NTT) in 2010 August (Titov et al. 2011) have now been tracked with the AuScope radio telescopes in 2011-2012.

In this paper, we continue our spectroscopic observations of the optical counterparts of southern IVS sources, in particular those with a long VLBI observational history. Some strong radio sources from the northern hemisphere have also been observed. Optical identifications were sought initially from the image and catalog data from the SuperCOSMOS Sky Surveys on the grounds of their small digitization pixel scale and excellent astrometric accuracy (Hambly et al. 2001). We also took advantage of the Sloan Digital Sky Survey (SDSS; York et al. 2000) DR8 in the regions where it was available. This improved the identification process, especially in regions of high stellar density.

In addition, we observed five weaker radio sources from the NVSS (NRAO VLA Sky Survey; Condon et al. 1998) and FIRST catalogs (Faint Images of the Radio Sky at Twenty cm; Becker et al. 1995) that we identified with objects from the SDSS whose colors were typical of high redshift quasars. Such high redshift quasars provide unique information about the early stages of the universe. The number of known radio sources with $z \geqslant 4$ is small, and we are exploiting this technique in an attempt to increase their number.

The observations and data reduction procedures are described in Section 2 and we report our results, along with detailed comments on individual objects, in Section 3.

\section{OBSERVATIONS}

Spectroscopic observations were carried out at five optical facilities.

ESO NTT. We had a five-night observing run in Visitor Mode at the European Southern Observatory (ESO) $3.58 \mathrm{~m}$ NTT at La Silla in 2011 December (088.A-0021 (A)) using the ESO Faint Object Spectrograph and Camera system with grism 13 covering the wavelength range 3685-9315 $\AA$. The seeing during observations was typically $0^{\prime \prime} .5-1^{\prime \prime} .5$, with a wavelength resolution 21.2 $\AA$. Exposure times varied from 10 minutes to $1 \mathrm{hr}$ depending on the magnitude of each target and current sky conditions. Wavelength calibration was performed using the spectra of an HeNeAr comparison lamp, resulting in an rms accuracy of $0.5 \AA$.

Gemini. A large number of targets were observed in Service Mode at the Gemini North and Gemini South $8.2 \mathrm{~m}$ telescopes through the Poor Weather Program (GS-2011A-Q-89, GN-2011B-Q-109, GS-2011A-Q-94) using the Gemini MultiObject Spectrograph system with grating R400. This grating covers $4500 \AA$ centered either at $5200 \AA$ or $6500 \AA$. As expected, the seeing and weather conditions were variable but the program overall was very successful. The wavelength resolution was $\sim 15 \AA$, and an exposure time of 20 minutes was used for all targets. Wavelength calibration was performed using the spectra of a CuAr lamp, resulting in an rms accuracy of $\sim 0.3 \AA$.

NOT. Observations with the $2.5 \mathrm{~m}$ Nordic Optical Telescope (NOT) on La Palma were carried out using the Andalucia Faint Object Spectrograph and Camera spectrograph, either with grism 5 or grism 4 with the WG345 blocking filter. The nominal wavelength range for grism 4 is $3200-9100 \AA$, with the second-order blocking filter cutting below $3560 \AA$. The red end of the detector suffers fringing, so the effective longwavelength limit is about $8000 \AA$. For grism 5 the nominal range is $5000-10250 \AA$. The slit width was $1^{\prime \prime} .0$ or $1^{\prime \prime} .3$ depending on the seeing. The typical integration time was between 20 and 40 minutes. The longer integrations were divided into two and the target was offset along the slit in order to improve the fringe correction. For the single integrations, internal halogen lamp images were taken before and after the science frame. Wavelength calibration was based on an HeNe lamp exposure taken before the science frame(s), resulting in an rms accuracy of $\sim 0.5 \AA$.

BTA. Two objects were observed in Visitor Mode at the $6 \mathrm{~m}$ Big Azimuthal Telescope (BTA) telescope of the Special Astrophysical Observatory in Russia in 2011 August, using the SCORPIO multi-mode focal reducer with GR300 grism covering the wavelength range 3500-9500 A. The seeing during observations was about $2^{\prime \prime}$. Spectral resolution was typically $20 \AA$.

Data reduction was performed with the IRAF software suite ${ }^{7}$ using standard procedures for spectral analysis. We removed the bias and pixel-to-pixel gain variations from each frame and then removed cosmic rays using the IRAF taskszAP. Where more than one exposure was obtained, the separate exposures were combined. Spectrum extraction, sky subtraction, and wavelength calibration were then carried out and the final onedimensional spectra were flux-calibrated with a spectrophotometric standard observed with the same instrumental setup. Because the conditions were often non-photometric, especially for observations made through the Gemini Poor Weather Program, the flux calibration should be taken as approximate.

\section{RESULTS}

Spectra of 120 IVS objects are shown in Figure 1, along with the line identifications. A blue, dashed line indicates lines that were used for redshift calculation, while a red, dot-dashed line indicates lines that were detected, generally at a low signal-tonoise ratio $(\mathrm{S} / \mathrm{N})$, but not used in determining the mean redshift.

Table 1 lists the IVS sources with their ICRF2 coordinates (which refer to the epoch J2000.0), the telescope used for each spectrum, the identified emission lines with their rest and observed wavelengths, the mean redshift and error, and brief notes on individual sources. More detailed notes on individual sources (indicated by an asterisk in the final column) are given in Section 3.2.

The quoted errors $\Delta z$ in the mean redshift $\bar{z}$ are given by

$$
\Delta z=\left\{\left[\left(\sigma_{z}\right)^{2}+\left(\Delta \lambda / \overline{\lambda_{0}}\right)^{2}\right] / N\right\}^{1 / 2}
$$

where $\sigma_{z}$ is the measured standard deviation among the independent estimates of $z, \Delta \lambda$ is the rms error in the wavelength calibration (typically $0.5 \AA$ ), and $\overline{\lambda_{0}}$ is the mean rest wavelength of the $N$ lines used to measure $\bar{z}$. Single-line redshifts (mostly $\mathrm{Mg}$ II) are assigned a conservative error of 0.001 if the $\mathrm{S} / \mathrm{N}$ is high and the line is symmetric. If the $\mathrm{S} / \mathrm{N}$ is low or the line is broad or asymmetric, then an (arbitrary) error of 0.002 is assigned; in two extreme cases where the $\mathrm{S} / \mathrm{N}$ is low and the line is broad or asymmetric (IVS B0633-26B and B1129-161), the redshift is given with a colon(:) appended and no error.

\footnotetext{
7 IRAF is distributed by the National Optical Astronomical Observatory, which is operated by the Association of Universities for Research in Astronomy, Inc., under contract to the National Science Foundation.
} 

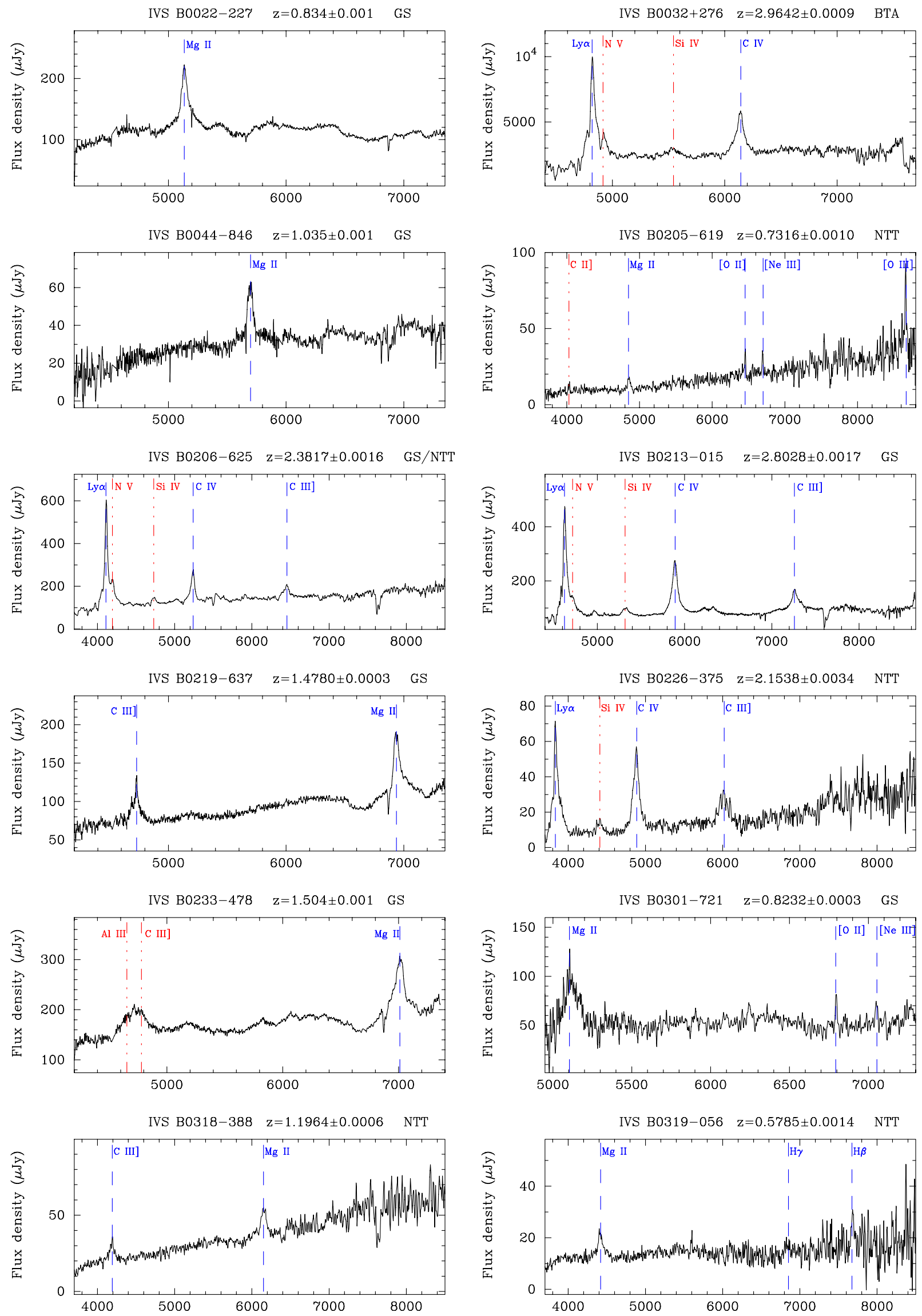

Figure 1. Optical spectra for 120 emission-line IVS targets. Dashed lines (blue) indicate emission lines used for redshift determination; dot-dashed lines (red) indicate lines detected at a lower signal-to-noise ratio or blended.

(An extended, color version of this figure is available in the online journal.) 
Table 1

Observed Emission Lines

\begin{tabular}{|c|c|c|c|c|c|c|c|c|}
\hline Source & R.A. $(\mathrm{J} 2000)^{\mathrm{a}}$ & Decl. $(\mathrm{J} 2000)^{\mathrm{a}}$ & Telescope $^{b}$ & Line & Rest $\lambda$ & Obs $\lambda^{\mathrm{c}}$ & $\bar{z}$ & Note $^{\mathrm{d}}$ \\
\hline IVS B0022-227 & 002524.2474 & -222747.596 & GS & Mg II & 2799.9 & 5136.3 & $0.834 \pm 0.001$ & $\mathrm{~S}$ \\
\hline \multirow[t]{4}{*}{ IVS B0032+276 } & 003443.4861 & +275425.721 & BTA & Ly $\alpha$ & 1215.7 & 4820.4 & & \\
\hline & & & & $\mathrm{Nv}$ & 1240.1 & [4921.7] & & \\
\hline & & & & Si IV & 1398.3 & [5545.9] & & \\
\hline & & & & C IV & 1549.5 & 6141.2 & $2.9642 \pm 0.0009$ & \\
\hline IVS B0044-846 & 004426.6883 & -842239.988 & GS & Mg II & 2799.9 & 5697.2 & $1.035 \pm 0.001$ & $\mathrm{~S} z=1.032[\mathrm{~S} 12]$ \\
\hline \multirow[t]{5}{*}{ IVS B0205-619 } & 020640.0029 & -614332.206 & NTT & $\mathrm{C}$ II] & 2326.9 & [4032.5] & & \\
\hline & & & & Mg II & 2799.9 & 4855.9 & & \\
\hline & & & & {$[\mathrm{O} \mathrm{II}]$} & 3726.8 & 6454.0 & & \\
\hline & & & & {$[\mathrm{Ne}$ III $]$} & 3868.7 & 6694.0 & & \\
\hline & & & & [O III] & 5006.8 & 8661.5 & $0.7316 \pm 0.0010$ & \\
\hline \multirow[t]{5}{*}{ IVS B0206-625 } & 020801.1713 & -621635.533 & GS/NTT & $\operatorname{Ly} \alpha$ & 1215.7 & 4114.9 & & \\
\hline & & & & $\mathrm{Nv}$ & 1240.1 & [4198.6] & & \\
\hline & & & & Si IV & 1398.3 & [4733.6] & & \\
\hline & & & & C IV & 1549.5 & 5237.6 & & \\
\hline & & & & $\mathrm{C}$ III] & 1908.7 & 6451.8 & $2.3817 \pm 0.0016$ & \\
\hline \multirow[t]{5}{*}{ IVS B0213-015 } & 021605.6638 & -011803.397 & GS & Ly $\alpha$ & 1215.7 & 4624.9 & & \\
\hline & & & & $\mathrm{Nv}$ & 1240.1 & {$[4720.2]$} & & \\
\hline & & & & Si IV & 1398.3 & {$[5326.7]$} & & \\
\hline & & & & C IV & 1549.5 & 5887.3 & & \\
\hline & & & & C III] & 1908.7 & 7261.7 & $2.8028 \pm 0.0017$ & \\
\hline \multirow[t]{2}{*}{ IVS B0219-637 } & 022054.1727 & -633019.387 & GS & C III] & 1908.7 & 4730.2 & & \\
\hline & & & & Mg II & 2799.9 & 6937.4 & $1.4780 \pm 0.0003$ & \\
\hline \multirow[t]{4}{*}{ IVS B0226-375 } & 022833.7343 & -371956.338 & NTT & $\operatorname{Ly} \alpha$ & 1215.7 & 3842.4 & & \\
\hline & & & & Si IV & 1398.3 & [4408.5] & & \\
\hline & & & & CIV & 1549.5 & 4881.0 & & \\
\hline & & & & C III] & 1908.7 & 6014.0 & $2.1538 \pm 0.0034$ & \\
\hline \multirow[t]{3}{*}{ IVS B0233-478 } & 023506.4235 & -473710.574 & GS & Al III & 1858.8 & [4692.1] & & \\
\hline & & & & $\mathrm{C}$ III] & 1908.7 & [4784.9] & & \\
\hline & & & & Mg II & 2799.9 & 7011.2 & $1.504 \pm 0.001$ & $S *$ \\
\hline \multirow[t]{3}{*}{ IVS B0301-721 } & 030138.4456 & -715634.399 & GS & $\mathrm{Mg}_{\text {II }}$ & 2799.9 & 5105.8 & & \\
\hline & & & & {$[\mathrm{O} \mathrm{II}]$} & 3726.8 & 6795.7 & & \\
\hline & & & & [Ne III $]$ & 3868.7 & 7051.2 & $0.8232 \pm 0.0003$ & \\
\hline \multirow[t]{2}{*}{ IVS B0318-388 } & 032046.4048 & -383728.503 & NTT & C III] & 1908.7 & 4191.0 & & \\
\hline & & & & Mg II & 2799.9 & 6151.3 & $1.1964 \pm 0.0006$ & \\
\hline
\end{tabular}

Notes.

a ICRF2 radio position.

b Telescope abbreviations: BTA = Big Azimuthal Telescope, GS = Gemini North, NOT = Nordic Optical Telescope, NTT = ESO New Technology Telescope.

${ }^{c}$ Square brackets indicate the line was not used for redshift determination.

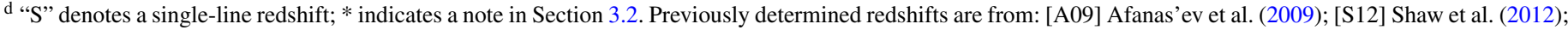
[S] Simbad database; [6dF] 6dF survey, Jones et al. (2009).

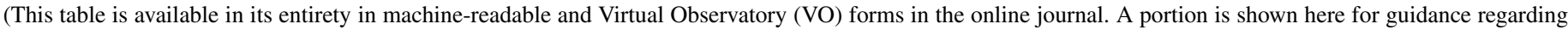
its form and content.)

Nineteen objects, listed in Table 2 with their ICRF2 positions (Fey et al. 2009), were found to have a good S/N (typically $\sim 60-110$ ) but featureless spectra, and hence are identified as probable BL Lac objects. Their spectra are shown in Figure 2.

A further 18 targets had spectra with an $\mathrm{S} / \mathrm{N}$ that was too low for confident spectral classification; these are listed in Table 3 with their ICRF2 positions.

There were five IVS targets that returned stellar spectra. This was assumed to be the result of foreground obscuration and, in most cases, small but significant offsets between SuperCOSMOS optical and ICRF2 radio positions. In two cases, the correct identification was found when the fields were reobserved in excellent seeing. Further discussion of these five objects is given in Section 3.1.

\subsection{Separation of Close Objects}

Occasionally, Galactic stars are found close on the sky to the radio position, leading to possible misidentification. Here we note several cases that were encountered during the observing runs.

1. IVS B0900-664-the spectrum obtained was that of a red M star, which is offset by 0'.52 from the ICRF position. A faint counterpart was seen in the NTT $B$-band acquisition image, but a much longer integration in good seeing will be needed to secure a redshift.

2. IVS B0905-202-the nearest object, as seen by SuperCOSMOS, was an $R=14$ m.6 stellar object located 1".55 from the radio position. Our NTT acquisition image, taken in 0'.6 seeing, showed a faint object at the radio position, but on the limb of the stellar disk. Spectroscopy of this faint $R \sim 22$ object did not reveal any clear emission lines.

3. IVS B1657-261-located in a very crowded star field in the Galactic bulge at a latitude of $b=9$.7. Identification of the optical counterpart may not be feasible.

4. IVS B1946-582-based on the SuperCOSMOS optical position, the radio minus optical position difference is only 

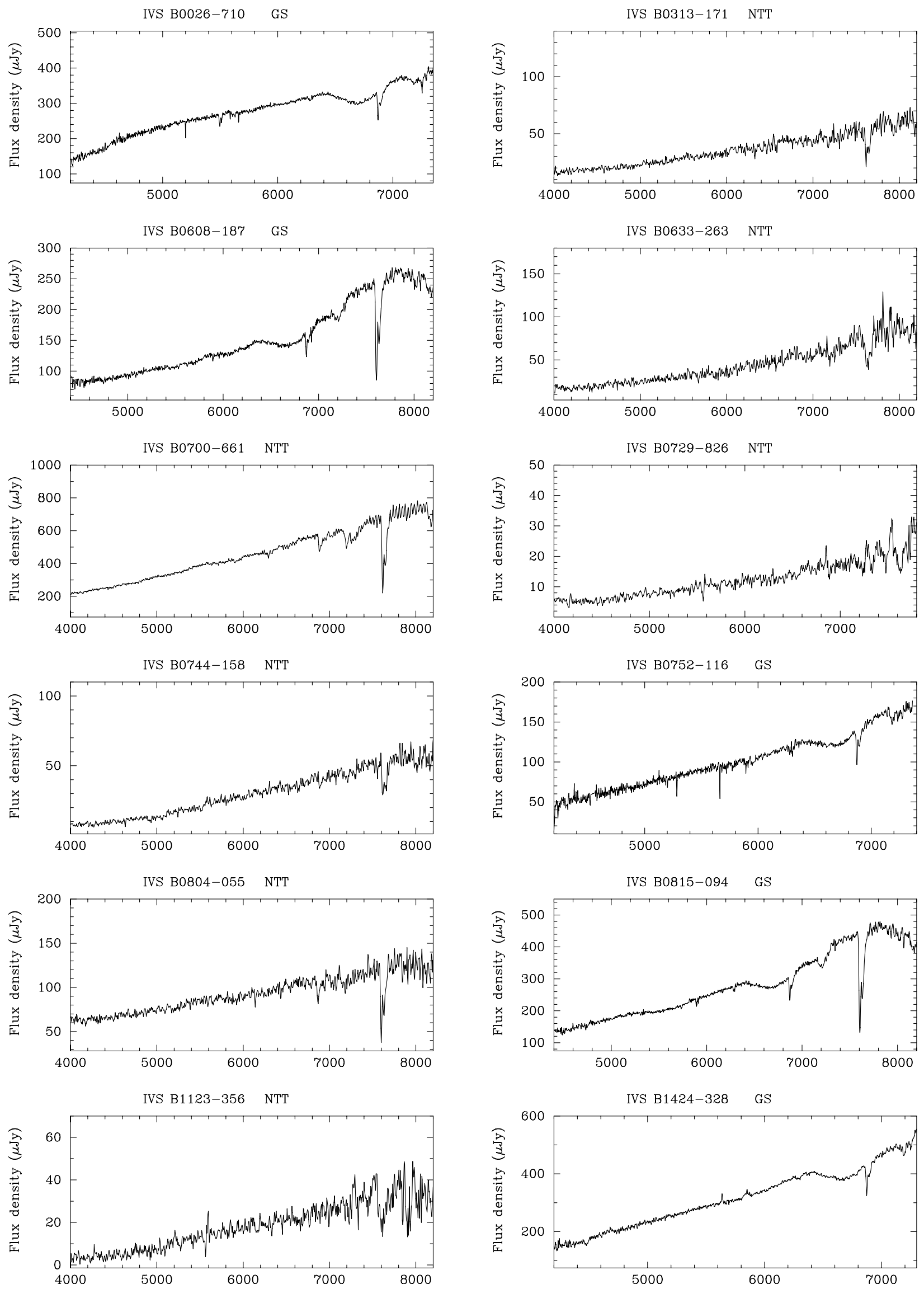

Figure 2. Spectra of 19 probable BL Lac objects from our observations, classified on the basis of their featureless spectra; see Table 2 for ICRF2 positions. 

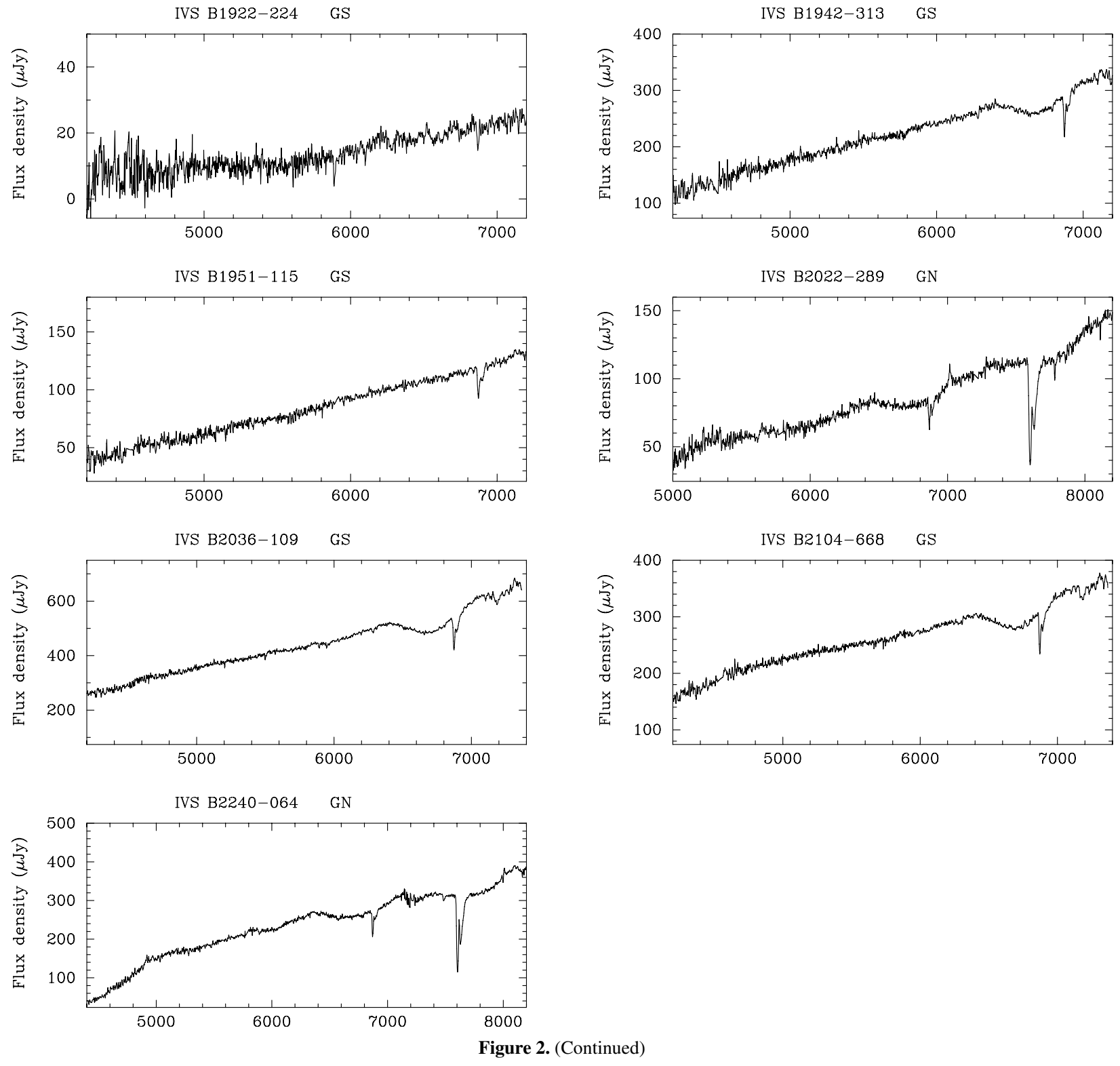

$0{ }^{\prime} 06$ in right ascension and $-0{ }^{\prime} 04$ in declination, but the Gemini South observation of this $R \sim 19$ object showed a typical stellar spectrum. The radio source has a flat spectrum with flux density 200-300 mJy at cm wavelengths.

5. IVS B2300-307-the obscuration of the optical field of this source by a foreground star was discussed previously (Titov et al. 2011). However, a later $80 \mathrm{~s}$ image in 0'.6 seeing (Figure 3) revealed a faint $R \sim 22$ object coincident with the radio position. Our spectroscopy yielded a redshift of $1.039 \pm 0.002$ based on weak C III] and Mg II emission.

\subsection{Notes on Individual Targets}

1. IVS B0233-478 - the broad feature attributed to Al III and C III] may also include Si III $\lambda 1890$.

2. IVS B0417-302 - prominent Fe II multiplet emission; intervening $\mathrm{Mg}$ II doublet absorption at $z_{\mathrm{abs}}=0.8395 \pm$ 0.0005 .

3. IVS B0447-507-possible double-peaked C III] line and prominent Fe II multiplet bands.

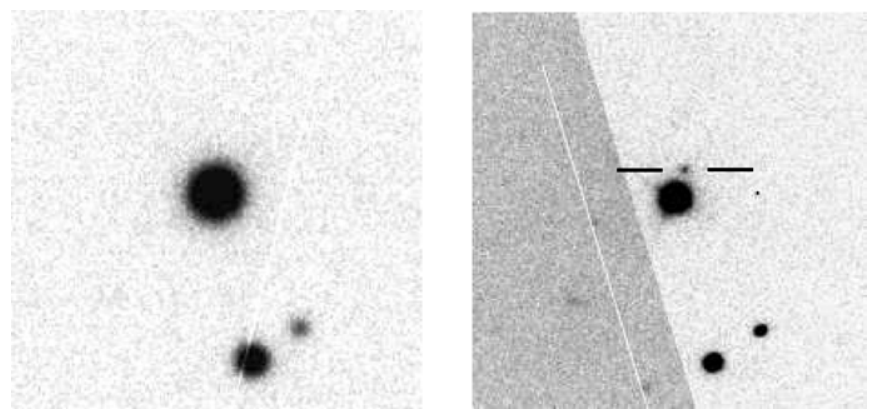

Figure 3. Two acquisition images of the sky field around the quasar IVS B2300-307 made at the NTT in 2010 August (left: seeing 2") and 2011 December (right: seeing 0.'6). Each image is $50^{\prime \prime}$ on a side; north is up and east to the left. The foreground star in the center of the left image completely obscures the quasar due to its large seeing disk. In the right image, the much better seeing reveals the faint quasar $(R \sim 22$, marked between the bars), separated by 3."7 from the star. (The diagonal step in contrast in the right image is an artifact of fast readout using two amplifiers for the acquisition image.)

4. IVS B0448-482-poor redshift match to C III] may indicate the presence of Si III $\lambda 1890$; prominent Fe II multiplet bands. 
Table 2

Probable BL Lac Objects

\begin{tabular}{llcc}
\hline \hline Source & R.A. $(\mathrm{J} 2000)^{\mathrm{a}}$ & Decl. $(\mathrm{J} 2000)^{\mathrm{a}}$ & Telescope \\
\hline IVS B0026-710 & 002841.5626 & -704515.926 & GS \\
IVS B0313-171 & 031527.6783 & -165629.711 & NTT \\
IVS B0608-187 & 061017.8861 & -184740.090 & GS \\
IVS B0633-263 & 063520.9090 & -262039.866 & NTT \\
IVS B0700-661 & 070031.2531 & -661045.232 & NTT \\
IVS B0729-826 & 072339.4063 & -824741.089 & NTT \\
IVS B0744-158 & 074618.2360 & -155534.745 & NTT \\
IVS B0752-116 & 075426.4564 & -114716.948 & GS \\
IVS B0804-055 & 080709.6175 & -054113.914 & NTT \\
IVS B0815-094 & 081749.7495 & -093330.528 & GS \\
IVS B1123-356 & 112531.4818 & -355703.352 & NTT \\
IVS B1424-328 & 142741.3610 & -330531.505 & GS \\
IVS B1922-224 & 192539.7901 & -221935.112 & GS \\
IVS B1942-313 & 194559.3693 & -311138.356 & GS \\
IVS B1951-115 & 195441.1557 & -112322.641 & GS \\
IVS B2022-289 & 202553.6128 & -284548.697 & GS \\
IVS B2036-109 & 203900.7104 & -104641.863 & GS \\
IVS B2104-668 & 210851.8207 & -663722.744 & GS \\
IVS B2240-064 & 224308.7606 & -060902.567 & GN \\
\hline
\end{tabular}

Note. ${ }^{\text {a }}$ ICRF2 radio position.

5. IVS B0521-262 - strong associated absorption in the Ly $\alpha$ emission line and a clear detection of the Lyman limit at the emission redshift.

6. IVS B0529-031 - faint optical counterpart with a very red spectrum, showing a single broad emission line identified as $\mathrm{Mg}$ II with associated $\mathrm{Mg}$ II absorption at $z_{\mathrm{abs}}=$ $1.8744 \pm 0.0004$, corresponding to a relative blue-shift of $\sim 1500 \mathrm{~km} \mathrm{~s}^{-1}$; a further absorption line at $8182.1 \AA$ remains unidentified. Given the low signal blueward of $6000 \AA$, together with the faint SDSS blue magnitudes ( $u=24.41, g=21.93)$, it is not surprising that $\left.\mathrm{C}_{\mathrm{III}}\right]$ and $\mathrm{C}$ IV are not detected.

7. IVS B0548-527-redshift in Table 1 is for the forbidden lines; the permitted lines of $\mathrm{Mg}$ II and $\mathrm{H} \beta$ show a redshift systematically higher by $830 \pm 80 \mathrm{~km} \mathrm{~s}^{-1}$.

8. IVS B0554+242-narrow self-absorption in Ly $\alpha$ and C IV at $z_{\mathrm{abs}}=3.2319 \pm 0.0010$.

9. IVS B0608-230-wavelengths for C III] and $\mathrm{C}_{\mathrm{IV}}$ are not consistent, suggesting the presence of additional lines or asymmetric structure; in addition, the Ly $\alpha$ wavelength is likely to be affected by absorption in the blue wing.

10. IVS B0633-26B - a very faint galaxy; a low S/N spectrum, with a single broad emission line, assumed to be $\mathrm{Mg}$ II.

11. IVS B0810-180 - very broad line wings in C IV and Ly $\alpha$, extending $\sim 30,000 \mathrm{~km} \mathrm{~s}^{-1}$ redward of the line peaks.

12. IVS B0828-064-poor consistency in redshift between $\mathrm{C}$ IV and $\mathrm{C}$ III], possibly due to associated absorption in the blue wing of $\mathrm{C} I V . \mathrm{Mg}$ II is present with a low $\mathrm{S} / \mathrm{N}$ just redward of the atmospheric $A$-band.

13. IVS B0844-557-redshift in Table 1 is for the forbidden lines; permitted lines $\mathrm{Mg}$ II and $\mathrm{H} \beta$ are displaced $\sim 1000 \mathrm{~km} \mathrm{~s}^{-1}$ to higher redshift.

14. IVS B0948-860 - single emission line, assumed to be Ly $\alpha$; the adopted redshift, $z=3.696$, is consistent with the $40 \%$ continuum depression blueward of the emission line due to the Ly $\alpha$ forest, and the possible detection of $\mathrm{C}$ IV at the red edge of the spectrum.
Table 3

Objects with Low Signal-to-noise Spectra

\begin{tabular}{llcc}
\hline \hline Source & R.A. $(\mathrm{J} 2000)^{\mathrm{a}}$ & Decl. $(\mathrm{J} 2000)^{\mathrm{a}}$ & Telescope \\
\hline IVS B0205-242 & 020733.3992 & -240202.210 & NTT \\
IVS B0227-542 & 022912.7856 & -540324.036 & NTT \\
IVS B0244-050 & 024649.1315 & -045110.933 & NTT \\
IVS B0253-754 & 025310.8816 & -751316.686 & GS \\
IVS B0334-546 & 033553.9248 & -543025.114 & NTT \\
IVS B0458+138 & 050145.2708 & +135607.220 & NTT \\
IVS B0516-621 & 051644.9261 & -620705.389 & NTT \\
IVS B0600+219 & 060351.5570 & +215937.698 & GN \\
IVS B0628-627 & 062857.4877 & -624844.744 & NTT \\
IVS B0705-649 & 070526.0526 & -650043.484 & NTT \\
IVS B0718-523 & 071914.2919 & -522832.506 & NTT \\
IVS B0821-094 & 082352.0233 & -093925.907 & NTT \\
IVS B0905-202 & 090754.0404 & -202649.475 & GS \\
IVS B0939-077 & 094221.4614 & -075953.204 & GS \\
IVS B1118+073 & 112038.4438 & +070447.174 & NTT \\
IVS B1134-739 & 113609.6597 & -741545.274 & NTT \\
IVS B1821-525 & 182513.8097 & -523058.281 & GS \\
IVS B2020-015 & 202332.8163 & -012342.153 & GS \\
\hline
\end{tabular}

Note. ${ }^{\text {a }}$ ICRF2 radio position.

15. IVS B0952-185-associated absorption at the emission redshift in both Ly $\alpha$ and C IV.

16. IVS B0956-409-Ly $\alpha$ is strongly self-absorbed.

17. IVS B1004-125-reported as having $z=0.24$ in Simbad (no reference given), clearly inconsistent with our Gemini spectrum; strong intervening heavy-element system at $z_{\mathrm{abs}}=1.5786 \pm 0.0002$ based on Fe II $\lambda \lambda 2344,2382,2586$, 2600 and $\mathrm{Mg}$ II $\lambda \lambda 2796,2803$ absorption.

18. IVS B1020+270 - extended red wing in C IV; C III] is not detected due to strong CCD fringing.

19. IVS B1039-474-Si IV, C IV and C III] emission lines all show strongly extended blue wings; redshift is based on the peak positions of the lines.

20. IVS B1127-443-single-line redshift is supported by stellar absorption features $(G$-band, $\mathrm{MgIb})$ noted in the plotted spectrum.

21. IVS B1143-696-spectrum is very similar to that of 3C 273, with strong Fe II multiplet emission in the region around $\mathrm{H} \beta$ (Wampler \& Oke 1967).

22. IVS B1722+562 - strong associated absorption system at $z_{\mathrm{abs}}=2.2463 \pm 0.0002$ seen in C IV, Si IV, N v and Ly $\alpha$. This has led to a relatively large uncertainty in emission redshift.

23. IVS B2235-556-strong self-absorption in the red wing of C IV.

24. IVS B2334-525-broad Mg II, possibly double-peaked with an extended red wing.

25. IVS B2341+295-very blue object based on POSS II sky survey images; rise in the spectrum redward of $7000 \AA$ may be due to the underlying galaxy or an unrelated object on the spectrograph slit. Intervening $\mathrm{Mg}$ II absorber at $z=0.8644 \pm 0.0004$.

\subsection{Spectra of Color-selected Quasars}

In an attempt to find more high-redshift radio quasars for our VLBI proper motion studies as a function of redshift, we selected five weak radio sources from the FIRST and NVSS catalogs for which the SDSS colors suggested the likelihood of $u$ - or $g$-band dropouts and the possibility of high redshifts. 
Table 4

SDSS Magnitudes and Redshifts for Five Color-selected NVSS Radio Sources

\begin{tabular}{|c|c|c|c|c|c|c|c|c|c|}
\hline Source & R.A. $(J 2000)^{a}$ & Decl. $(\mathrm{J} 2000)^{\mathrm{a}}$ & $g$ & $r$ & Telescope $^{b}$ & Line & Rest $\lambda$ & $\operatorname{Obs} \lambda^{\mathrm{c}}$ & $\mathrm{z}^{\mathrm{d}}$ \\
\hline \multirow[t]{2}{*}{ NVSS J101335+281118 } & 101335.49 & +281118.3 & 25.76 & 22.03 & NOT & Ly $\alpha$ & 1215.7 & 6990 & \\
\hline & & & & & & $\operatorname{Ly} \beta$ & 1025.7 & 5897 & 4.75: \\
\hline NVSS J125944+240707 & 125944.59 & +240707.9 & 22.94 & 21.82 & NOT & $\mathrm{Mg}_{\text {II }}$ & 2799.9 & 5895.5 & $1.138 \pm 0.001$ \\
\hline NVSS J145459+110928 & 145459.36 & +110928.5 & 26.09 & 22.67 & NOT & Ly $\alpha$ & 1215.7 & 7208 & 4.93: \\
\hline NVSS J162830+115403 & 162830.55 & +115403.5 & 25.27 & 22.25 & NOT & $\mathrm{Ly} \alpha$ & 1215.7 & 6650 & 4.47: \\
\hline \multirow[t]{4}{*}{ NVSS J163400+640821 } & 163400.47 & +640821.6 & $24.67^{\mathrm{e}}$ & $19.57^{\mathrm{e}}$ & NOT & $\mathrm{Ly} \alpha$ & 1215.7 & [5628.4] & \\
\hline & & & & & & O I/Si II & 1303.3 & [6039.0] & \\
\hline & & & & & & Si IV & 1398.3 & [6458.1] & \\
\hline & & & & & & C IV & 1549.5 & 7157.7 & $3.619 \pm 0.001$ \\
\hline
\end{tabular}

Notes.

a NVSS radio position.

b Nordic Optical Telescope.

c Square brackets indicate the line was not used for redshift determination.

${ }^{\mathrm{d}}$ Colon indicates approximate redshift based on $\operatorname{Ly} \alpha$ alone.

e Magnitudes are $u$ and $g$, respectively, for this object.

Spectra were obtained at the NOT on the nights of 2012 May 24-26. The SDSS colors, wavelengths, and redshifts for the five color-selected radio quasars are given in Table 4 . The "bluest" of the five, NVSS J125944+240707, was a $z=1.139$ radio galaxy, but the other four proved to be broad emission-line quasars with redshifts in excess of 3.5, including three with $z>4$. This is an excellent return for our search and a striking result from the NOT, the smallest of the telescopes used in this program. The five spectra are shown in Figure 4 and further information is given in Table 4 . Redshifts for the $z>4$ quasars are based on estimated wavelengths for $\operatorname{Ly} \alpha$ (and, in one case, $\operatorname{Ly} \beta$ ) and are very uncertain because of strong absorption in the blue wing of the line.

A comparison between the FIRST and NVSS flux densities at $1.4 \mathrm{GHz}$ for the five sources showed that one source, NVSS $\mathrm{J} 145459+110928$, appeared to show evidence of variability, with $S_{\mathrm{NVSS}}=9.8 \pm 0.5 \mathrm{mJy}$ and $S_{\text {FIRST }}=15.07 \pm 0.14 \mathrm{mJy}$. This is also the only source that is unresolved in FIRST. The other four sources all show minor extension at the $1^{\prime \prime}$ level.

\section{REDSHIFT DISTRIBUTIONS}

Together with the 31 redshifts reported in Titov et al. (2011), we have now accumulated over 150 redshifts for IVS sources, mostly in the south. Their optical counterparts are systematically fainter than those in the Titov \& Malkin (2009) compilation. Since the IVS selection process is directed at compact, milliarcsecond radio quasars, it is important to test whether their redshift distribution differs from that of the FIRST-SDSS quasar sample (Kimball et al. 2011), which is selected without regard to morphology, spectral index, or angular size, and extends to much lower flux densities.

The redshift distribution of the sources from this paper and Titov et al. (2011) is shown in Figure 5(a). For comparison, Figure 5(b) shows the distribution of 1594 point sources with known redshifts from ICRF2, using redshift data from Titov $\&$ Malkin (2009). The SuperCOSMOS Hambly et al. (2001) morphological classification was used to remove galaxies from the Titov \& Malkin (2009) list, and the sample was further restricted to those with radio-optical offsets $<1^{\prime \prime}$ and Galactic latitudes $>10^{\circ}$ (A. L. Schaefer et al. 2013, in preparation).

The redshift distribution for the sources from this paper and the earlier paper (Titov et al. 2011) is completely consistent with having been drawn from the same redshift distribution as FIRST-SDSS quasars (Kimball et al. 2011), with a
Kolmogorov-Smirnov (K-S) test giving a probability $p=0.84$ of the two samples being drawn from the same distribution. Thus the sharp drop-off that we see in the number of quasars at $z \gtrsim 2$ is a reflection of the underlying quasar distribution, and not a selection effect of the IVS candidate sources. While the K-S plot for the ICRF2 sample shows an apparent excess of low-redshift sources compared with FIRST-SDSS, the difference is not significant, with a K-S probability of $p=0.24$. We attribute this excess to selection effects arising from the redshifts in (Titov \& Malkin 2009) being drawn from the literature, and therefore likely to be biased toward brighter, lower-redshift quasars.

\section{SUMMARY AND CONCLUSION}

We present redshifts and spectra for 120 emission-line objects identified with radio sources from the candidate International Celestial Reference Catalog. Most of the target objects are in the south and many had not previously been optically identified. While redshifts were usually based on two or more lines, those for 22 objects were based on a single emission line, in most cases assumed to be $\mathrm{Mg}$ II; many of these single-line redshifts were supported by other spectral information and most are considered reliable. In addition, we classed 19 sources as probable BL Lac objects, based on a high $\mathrm{S} / \mathrm{N}$ but featureless spectra. A further 18 targets were considered to have an $\mathrm{S} / \mathrm{N}$ too low for confident spectral classification.

The distribution of redshifts from this paper, together with those from our earlier paper (Titov et al. 2011), is consistent with the much larger sample drawn from FIRST and SDSS (Kimball et al. 2011). This implies that the ultra-compact, flatspectrum sources that make up the IVS Reference Catalog are not distinguished from the radio quasar population at large. On the other hand, the distribution of redshifts from the much larger sample drawn from ICRF2 (Titov \& Malkin 2009) has a small, but not significant excess of low-redshift quasars, almost certainly the result of observational selection.

This paper is based on observations collected at five telescopes:

1. ESO NTT, under the European Organisation for Astronomical Research in the Southern Hemisphere, Chile under program 088.A-0021(A).

2. Two Gemini Observatories, which are operated by the Association of Universities for Research in Astronomy, Inc., under a cooperative agreement with the National Science 

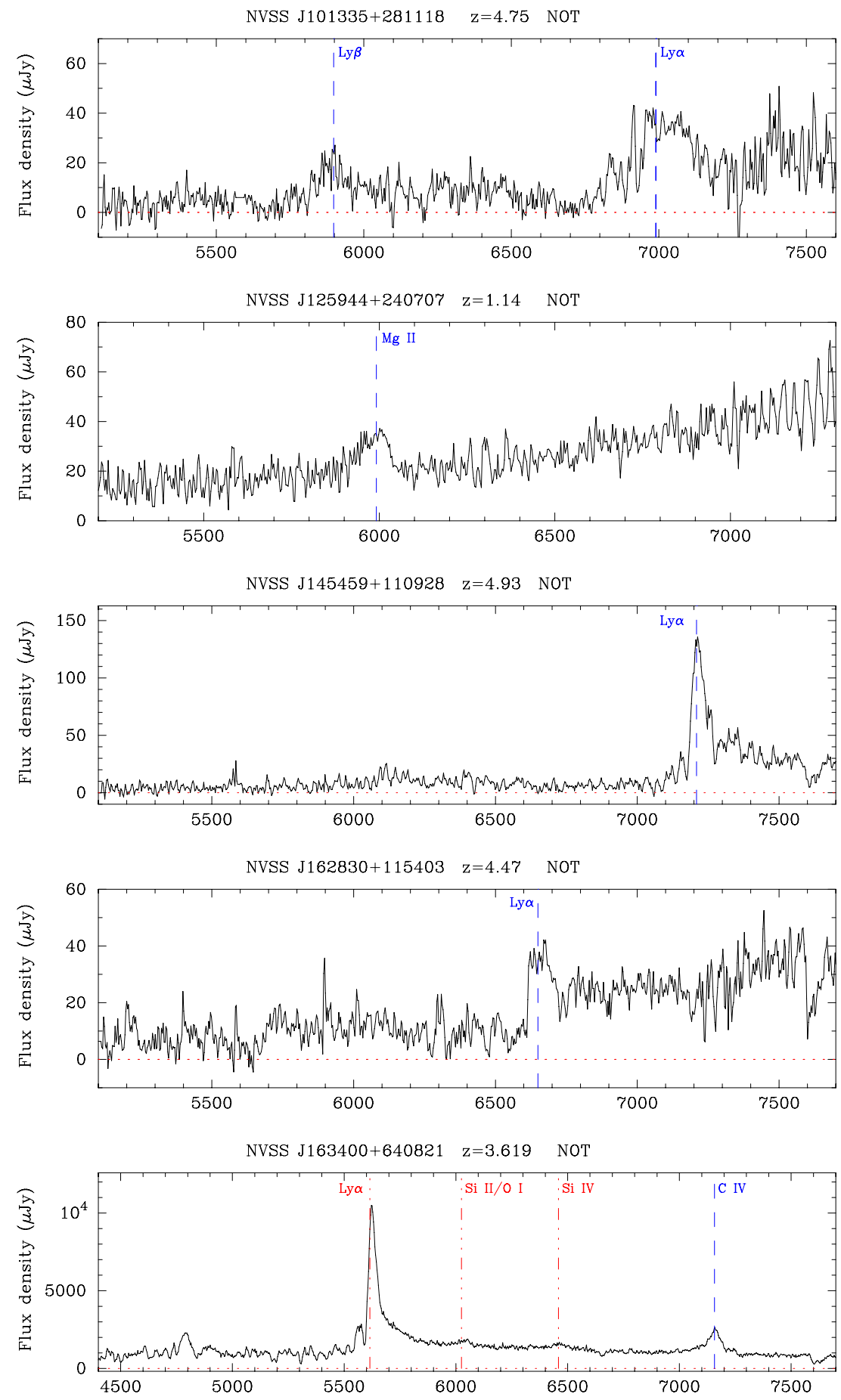

Figure 4. Spectra of the five color-selected quasars from NVSS/FIRST and SDSS. Dashed lines (blue) indicate emission lines used for redshift determination; dot-dashed lines (red) indicate lines detected at a lower signal-to-noise ratio or blended. Further details are given in Table 4.

(A color version of this figure is available in the online journal.)

Foundation on behalf of the Gemini partnership: the National Science Foundation (United States), the Science and Technology Facilities Council (United Kingdom), the National Research Council (Canada), CONICYT (Chile), the Australian Research Council (Australia), Ministurio da Ciuncia, Tecnologia e Inovacio (Brazil) and Ministerio de Ciencia, Tecnologia e Innovacion Productiva (Argentina) under programs GS-2011A-Q-89 and GS-2011B-Q-94 (Gemini South), and GN-2011B-Q-109 (Gemini North).
3. Six meter Big Azimuthal Telescope (BTA) operated by the Special Astrophysical Observatory (Russia).

4. Nordic Optical Telescope, operated on the island of La Palma jointly by Denmark, Finland, Iceland, Norway and Sweden, in the Spanish Observatorio del Roque de los Muchachos of the Instituto de Astrofisica de Canarias.

Two of us, Titov and Jauncey, were supported by a travel grant from the Australian Nuclear Science Technology Organisation (ANSTO) in their Access to Major Research Facilities Program 


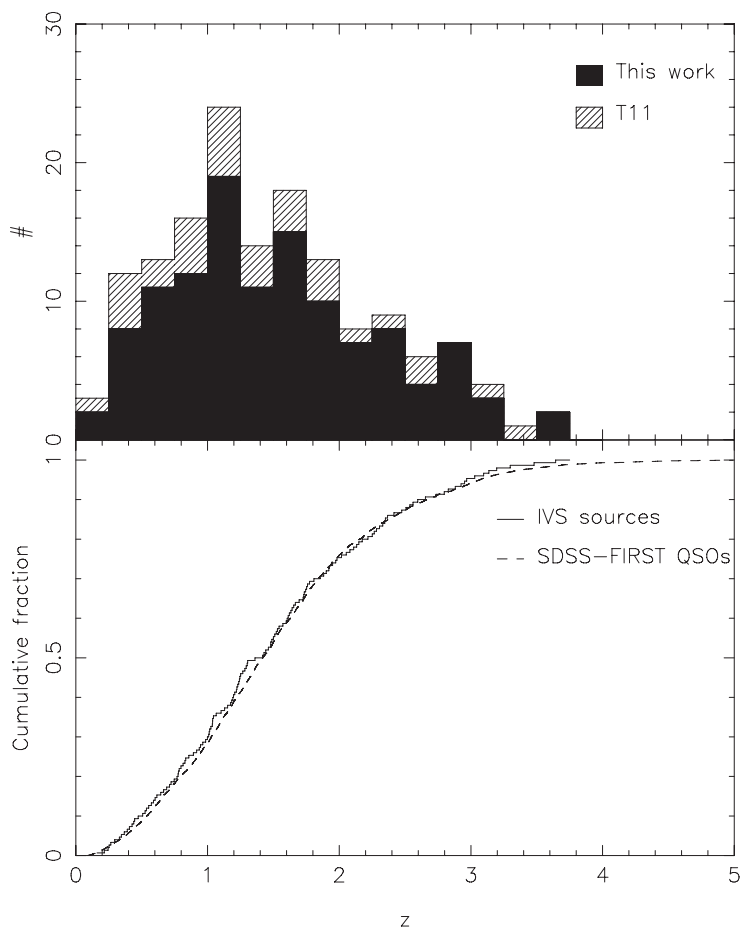

(a)

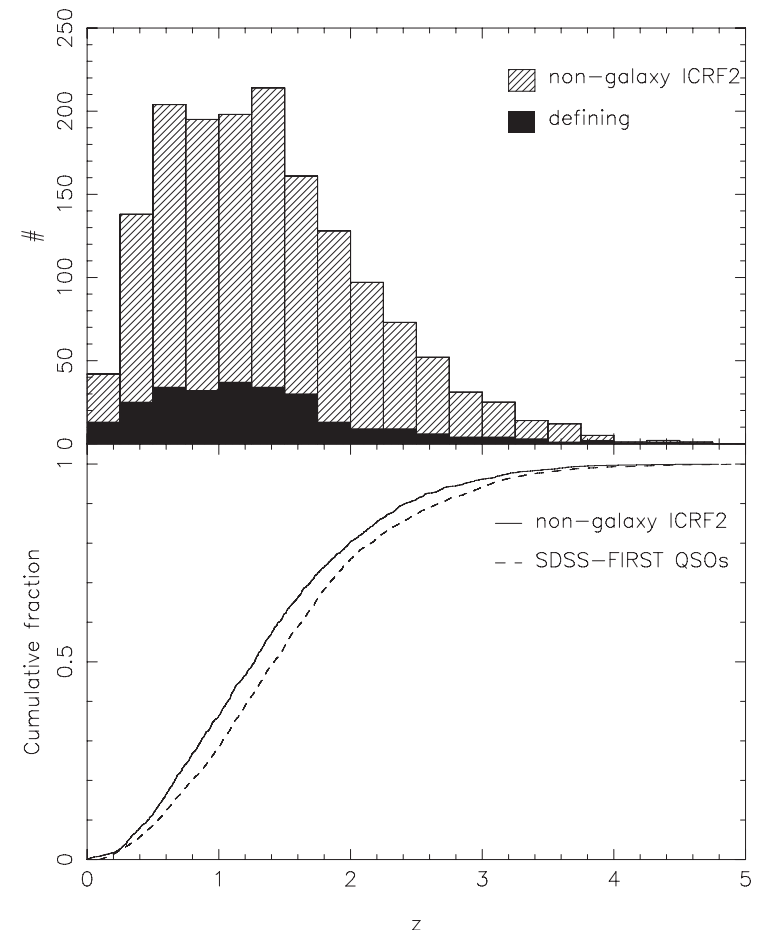

(b)

Figure 5. (a) Distribution of redshifts among the $\sim 150$ IVS sources from this paper and the previous paper (Titov et al. 2011, abbreviated above as T11). The lower panel shows the cumulative redshift distribution (K-S test) compared with the FIRST-SDSS sample (Kimball et al. 2011). (b) Corresponding redshift distribution and K-S test for 1594 ICRF2 quasars from Titov \& Malkin (2009); see text.

(AMRFP) (reference number AMRFP 10/11-O-31) to travel to the BTA telescope in Russia.

Funding for SDSS-III has been provided by the Alfred P. Sloan Foundation, the Participating Institutions, the National Science Foundation and the U.S. Department of Energy Office of Science. The SDSS-III Web site is http://www.sdss3.org/.

SDSS-III is managed by the Astrophysical Research Consortium for the Participating Institutions of the SDSS-III Collaboration including the University of Arizona, the Brazilian Participation Group, Brookhaven National Laboratory, University of Cambridge, Carnegie Mellon University, University of Florida, the French Participation Group, the German Participation Group, Harvard University, the Instituto de Astrofisica de Canarias, the Michigan State/Notre Dame/JINA Participation Group, Johns Hopkins University, Lawrence Berkeley National Laboratory, Max Planck Institute for Astrophysics, Max Planck Institute for Extraterrestrial Physics, New Mexico State University, New York University, Ohio State University, Pennsylvania State University, University of Portsmouth, Princeton University, the Spanish Participation Group, University of Tokyo, University of Utah, Vanderbilt University, University of Virginia, University of Washington, and Yale University.

AuScope is funded under the National Collaborative Research Infrastructure Strategy (NCRIS), an Australian Commonwealth Government Programme.

This paper is published with the permission of the CEO, Geoscience Australia.

\section{REFERENCES}

Afanas'ev, V. L., Dodonov, S. N., Moiseev, A. V., et al. 2009, ARep, 53, 287

Arias, E. F., Charlot, P., Feissel, M., \& Lestrade, J.-F. 1995, A\&A, 303, 604

Becker, R. H., White, R. L., \& Helfand, D. J. 1995, ApJ, 450, 559

Condon, J. J., Cotton, W. D., Greisen, E. W., et al. 1998, AJ, 115, 1693

de Bruijne, J. H. J. 2012, Ap\&SS, 341, 31

Fey, A., Gordon, G., \& Jacobs, C. (ed.) 2009, The Second realization of the International Celestial Reference Frame by Very Long Baseline Interferometry, IERS Technical Notes 35, Verlad des Bundesamts fur Kartographie und Geodasie, Frankfurt am Main

Hambly, N. C., Davenhall, A. C., Irwin, M. J., \& MacGillivray, H. T. 2001, MNRAS, 326, 1315

Jones, D. H., Read, M. A., Saunders, W., et al. 2009, MNRAS, 399, 683

Kimball, A. E., Ivezic, Z., Wiita, P. J., \& Schneider, D. P. 2011, AJ, 141,182

Ma, C., Arias, E. F., \& Eubanks, T. M. 1998, AJ, 116, 516

MacCarthy, D., \& Petit, G. (ed.) 2004, IERS Conventions (2003), IERS Technical Notes 32, Verlad des Bundesamts fur Kartographie und Geodasie, Frankfurt am Main

Mignard, F., \& Klioner, S. 2012, A\&A, 547, A59

Perryman, M. A. C., de Boer, K. S., Gilmore, G., et al. 2001, A\&A, 369,339

Schlüter, W., \& Behrend, D. 2007, JGeod, 81, 379

Shaw, M. S., Romani, R. W., Cotter, G. H., et al. 2012, ApJ, 748, 49

Titov, O., Dickey, J. M., Lovell, J. E. J., \& McCulloch, P. M. 2013, Proc. IAG Symp. 139, Earth on the Edge: Science for a Sustainable Planet, ed. C. Rizos \& P. Willis (Berlin: Springer)

Titov, O., Jauncey, D. L., Johnston, H. M., et al. 2011, AJ, 142, 165

Titov, O., \& Malkin, Z. 2009, A\&A, 506, 1477

Wampler, E. J., \& Oke, J. B. 1967, ApJ, 148, 695

York, D. G., Adelman, J., Anderson, J. E., Jr., et al. 2000, AJ, 120, 1579 